\title{
Lack of Guidelines for use of Inhaled Nitric Oxide in Preterm Infants Less Than 34 weeks Gestational Age
}

Dilip Bhatt, MD1, Rangasamy Ramanathan,MD². ${ }^{1}$ Southern California Kaiser Permanente, Fontana, ${ }^{2}$ LAC+USC Medical Center, Keck School of Medicine of USC, Los Angeles, California, USA.

\section{Background}

- The National Institute of Health (NIH) consensus panel in 2011 and Committee on Fetus and Newborn (COFN) of American Academy of Pediatrics (AAP) in 2014 concluded that the use of inhaled nitric oxide (iNO) in preterm infants $<34$ weeks gestational age (PTIs) is not recommended. Despite these recommendations, iNO use in Level III and Level IV NICUs is steadily increasing every year in California, USA. There are no published guidelines for iNO use in PTIs. We studied if there were any consistent patterns with iNO use in PTIs in the 4 Level III NICUs from a large Health Maintenance Organizations.

Methods

Retrospective review of prospectively entered data in PTIs with iNO treatment during their NICU stay and to describe the variations before, during or after initiation of iNO Rx.

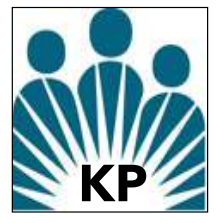

\section{Results}

- We found the following variations and or lack of documentation before, during or after iNO therapy in PTIs: 1. baseline oxygenation index or oxygen saturation index, 2. informing parents about off-label use of iNO, 3. using high frequency ventilation to optimize lung recruitment, 4. monitoring pre-and post-ductal oxygen saturation by pulse oximeter ( $\mathrm{SpO} 2), 5$. weaning iNO based on $\mathrm{SpO} 2$ rather than $\mathrm{PaO} 2,6$. echocardiogram to assess for pulmonary hypertension, 7. evaluation to rule out sepsis, 8 . targeting a certain hematocrit to improve oxygen delivery.

\section{Conclusions}

Significant variations exist regarding indications, work up and follow up before or during iNO treatment in PTIs. There is an urgent need to provide management guidelines to both Level III and Level IV NICUs. Providing guidelines based on the results from randomized, controlled clinical trials may decrease unnecessary use of iNO, and potentially decrease adverse effects and cost in this high-risk population.

\section{Future Directions}

- We propose the following minimum criteria prior to starting iNO Rx in PTIs: 1 . OI $\geq 20,2$. Pre- and post-ductal $\mathrm{SpO}_{2}>5 \%$, 3. Patient on HFOV, 4. Echocardiographic evidence for PPHN, such as, tricuspid regurgitation jet gradient $>36 \mathrm{~mm} \mathrm{Hg}$ or presence of septal flattening in systole.

- Regarding starting dose of iNO, 10-20 ppm may be appropriate. Response to iNO Rx using pre-ductal $\mathrm{SpO}_{2}$ between $88-92 \%$. Oxygen delivery may be optimized by keeping hematocrit $>35 \%$.

- Establish iNO Registry and record detailed data from all PTIs on iNO Rx 